\title{
Avaliação da atividade funcional em idosos submetidos à cinesioterapia em solo
}

\author{
Functional activity evaluation in elderly people after cinesiotherapy
}

\section{Aline Cristina Tavares ${ }^{1}$, Tatiana Sacchelli²}

\section{RESUMO}

Introdução. O declínio funcional está fortemente associado ao envelhecimento do indivíduo. Evidencia-se que o sedentarismo, a perda de massa e força muscular, a diminuição do equilíbrio, de propriocepção e de mobilidade desencadeiam a perda de independência. Objetivo. Verificar se uma intervenção fisioterapêutica baseada em exercícios de alongamentos, fortalecimento e equilíbrio pode proporcionar a melhoria do desempenho funcional de idosos acima de 65 anos. Método. 17 idosos com média de idade de 72,52 $\pm 4,65$ anos participaram do programa fisioterapêutico, realizado individualmente durante vinte e quatro sessões, sendo duas delas realizadas semanalmente com sessenta minutos de duração nas locações da Clínica de Fisioterapia da UMESP. Os dados foram colhidos com o questionário estruturado, versão brasileira do OARS: Brazilian Multidimensional Functional Assessment Questionnaire (BOMFAQ). Resultados. Apenas uma das atividades (andar no plano) apresentou melhora significativa (de $4,71 \pm 1,21$ para $5,65 \pm 0,79$ ) com $p=0,03$. As demais atividades não apresentaram alterações significativas. Conclusão. Constatou-se que houve a manutenção de todas as atividades funcionais após a intervenção fisioterapêutica.

Unitermos. Envelhecimento, Exercício, Força Muscular, Equilíbrio.

Citação. Tavares AC, Sacchelli T. Avaliação da atividade funcional em idosos submetidos à cinesioterapia em solo.

Trabalho realizado na Universidade Metodista de São Paulo. Clínica Escola-Modelo. São Bernardo do Campo-SP, Brasil

1. Pós-Graduanda em Fisioterapia Hospitalar na Universidade Metodista de São Paulo - UMESP, São Bernardo do Campo-SP, Brasil.

2. Fisioterapeuta, Mestre em Ciência do Movimento, Professora do Curso de Graduação em Fisioterapia da UMESP, São Bernardo do Campo-SP, Brasil.

\section{SUMMARY}

Introduction. Functional decrease is strongly associated with aging. It's shown that sedentarism, loss of muscle strength, muscle mass, balance, proprioception and mobility cause dependence. Objective. Verify whether physical therapeutically intervention based on stretching, strengthening and balance are to improve functional performance of elderly above 65 years of age. Method. 17 elderly at $72.45 \pm 4.65$ year's age participated in the program that was provided in 24 sections, 60 minutes each, twice a week. It took place in the department of physiotherapy of the São Paulo Methodist University (UMESP). The data were gathered with a Brazilian version of OARS, Brazilian Multidimensional Functional Assessment Questionnaire. Results. Only one activity (walking on the plane) has shown significant improvement (from $4.71 \pm 1.21$ to $5.65 \pm 0.79$ ) with $\mathrm{p}=0.03$. Conclusion. There was an upkeep of all activities after the physiotherapy intervention.

Keywords. Aging, Exercise, Muscle Strength, Balance.

Citation. Tavares AC, Sacchelli T. Functional activity evaluation in elderly people after cinesiotherapy. 


\section{INTRODUÇÃO}

Estima-se que em 2025 o Brasil terá mais do que o dobro do número de idosos existente hoje1. $\mathrm{E}$, uma vez que é um processo natural que possui as propriedades de ser universal, decremental, progressivo e intrínseco ${ }^{1,2}$, ocasionado por ação de genes implicados na síntese protéica que determinam a morte programada das células, e pela apoptose que promovem o declínio da atividade, alterações na estrutura e função dos órgãos celu$\mid a r^{1,3}$, ocorrem a diminuição do metabolismo em $3 \%$ a cada dez anos; a diminuição da capacidade aeróbica máxima e da tolerância ao exercício físico; a redução da velocidade de reflexos em $2 \%$ a cada ano; a diminuição da coordenação; o aumento da fragilidade e porosidade dos ossos; o adelgamento e tendência à degeneração das cartilagens; a diminuição do equilíbrio; a diminuição da massa muscular (sarcopenia) e a diminuição de força muscu$\operatorname{lar}^{1,4-12}$.

O declínio funcional pode ser atribuído à diminuição da acuidade visual, de funções do SNC (sistema nervoso central) ou de diminuição de força muscular, da flexibilidade, da propriocepção e deterioração do controle postural principalmente pelas alterações nas fibras musculares tipo II, do tipo de medicamento que se faz uso $0^{6,13,14}$, e da própria inatividade ${ }^{1,15}$. Assim, a dependência nas realizações das AVD's (atividades de vida diária) aumenta de três a cinco vezes de acordo com o aumento da inatividade ${ }^{16}$.

Partindo do princípio que todas as tarefas requerem um limiar da força para serem desempenhadas e que o controle postural proporciona meIhor estabilidade, maior rapidez e mobilidade durante o desenvolvimento delas, qualquer redução da massa, da força muscular ou do equilíbrio implica no prejuízo da performance nas AVD's $\mathrm{s}^{4,6,8,9,17}$, e o exercício físico pode melhorá-los além de diminuir os níveis de pressão arterial e melhorar o débito cardíaco, bem como retardar, senão reverter, as alterações relacionadas com a idade na função sináptica e na velocidade de condução nervosa ${ }^{9}$, aumentar o bem-estar e abaixar o nível de medo de cair e ansiedade, resultando em proteção contra queda e fratura e preservação das habilidades funcionais do idoso ${ }^{9,18,19}$.

O emprego do programa cinesioterapêutico que se baseie em exercícios de força muscular, flexibilidade e equilíbrio pode melhorar a resistência e a potência da funcionalidade de forma geral ${ }^{5,20} \mathrm{e}$ se mostra eficaz na melhora significativa do equilíbrio estático e dinâmico nos idosos, diminuindo o risco de quedas 16 e melhorando a qualidade de vida. Esses aspectos são de extrema importância, pois a duração do período de morbidade tem implicações sociais, pessoais e médicas de amplas dimensões. Para o indivíduo representa sofrimento psicológico e físico e para a sociedade representa falência devido os altos custos com os serviços sociais e de saúde ${ }^{13,20}$.

O objetivo do trabalho foi avaliar a melhora da atividade funcional em idosos submetidos à cinesioterapia.

\section{MÉTODO \\ Amostra}

Foi realizado um estudo transversal, na Clínica de Fisioterapia da Universidade Metodista de São Paulo (UMESP), após liberação do Comitê de Ética e Pesquisa tendo como critérios de inclusão: indivíduos acima de 64 anos de idade, sedentários, que compreendessem e assinassem o termo para participar do estudo, e como critérios de exclusão: pacientes com doenças neurológicas ou cadeirantes.

A avaliação e follow-up da amostra foram realizados por um profissional não envolvido diretamente com o estudo, a fim de evitar resultados tendenciosos.

\section{Procedimento}

A intervenção fez uso de 2 sessões semanais, com duração de 60 minutos cada. O tempo total de tratamento foi de 24 sessões (12 semanas). 0 programa de tratamento foi de exercícios para aquecimento em 5 minutos, exercício de alongamento dos músculos antigravitacionais por $6 \mathrm{mi}-$ nutos, exercícios de fortalecimento dos músculos antigravitacionais por 10 minutos, exercícios de equilíbrio por 25 minutos e 4 minutos finais para relaxamento muscular: 1) Aquecimento: andar de frente com passos amplos e com movimentos alternado de flexão de ombro de $90^{\circ}$ por $1 \mathrm{~min}$. Andar de frente realizando flexão de $90^{\circ}$ de quadril e de joelho alternando movimentos de membros superiores por $1 \mathrm{~min}$. Andar de lado com passos amplos, com movimento lateral de membros superiores, segurando um bastão em cada mão por 1 min para cada lado. Jogar bola com o terapeuta por 1 minuto. 2) Exercícios de alongamento dos músculos antigravitacionais: de extensores de jo- 
elho por 1 de cada lado, de extensores de quadril por 1 min de cada lado, alongamento de extensores de tronco por 1 min de cada lado. 3) Exercícios de fortalecimento dos músculos antigravitacionais (duração de $2 \mathrm{~min} 30 \mathrm{~s}, 1 \mathrm{~min}$ e $15 \mathrm{~s}$ de cada lado) dos músculos extensores de quadril, extensores de tronco superior, extensores de joelho, extensores de tronco inferior. 4) Exercícios de equilíbrio (nos quais todos os exercícios eram realizados com instabilizações do paciente pelo quadril e esperar que ele apresente reação de equilíbrio, e garantir maior segurança) com exercício sentado na bola sem aproximar os pés da bola e ou apoiar as mãos sobre a bola ou coxas, de modo que não se fixe, exercício sentado na bola tocando, na diagonal, uma das mãos o espaguete, exercício com a prancha de equilíbrio, deambulação sobre o espaguete, exercício com oscilação anterior realizada pelo fisioterapeuta, exercício com oscilação posterior realizada pelo fisioterapeuta, com apoio de antebraço na região anterior e posterior do tronco do paciente, exercício com mini-tramping em ortostatismo com apoio unilateral, exercício em pé com apoio em retropé, exercício com mini-tramping jogando bola com o fisioterapeuta, exercício no balancim. 5) Relaxamento por 1 min cada exercício - tração de cervical, tração lombar, fricção, massagem com bolinha.

O desfecho principal foi verificar a melhoria da atividade funcional, utilizando um questionário estruturado, versão brasileira do OARS: Brazilian Multidimensional Functional Assessment Questionnaire (BOMFAQ) ${ }^{21}$, validado para avaliar o desempenho funcional do idoso.

Com relação à pontuação, os valores foram estipulados de acordo com o auto-relato de cada participante, sendo 0 (zero) quando ele referia não realizar uma determinada atividade resultante de uma dificuldade muito intensa ou não saber realiza-la, 2 (dois) quando essa dificuldade era muita, 4 (quatro) quando pouca e 6 (seis) quando ausente.

\section{Análise Estatística}

Para a comparação dos resultados antes e depois do tratamento foi utilizado o teste não paramétrico Kruskal Wallis e para discriminar as diferenças foi utilizado o teste $t$ da Student. Os dados foram analisados no programa STATISTICA. Foi considerado um nível de significância de 5\% $(p<0,05)$.

\section{RESULTADOS}

Participaram do estudo 17 idosos com idade média de $72,52 \pm 4,65$ anos. Destes $88 \%$ eram do sexo feminino ( 15 participantes) e $12 \%$ do masculino (2 pacientes). Não houve desistências durante o tempo de intervenção.

Os valores dos dados de cada paciente conforme avaliação e comparados em pré e pós-tratamento encontram-se na Tabela 1. As atividades referidas representam a habilidade em deitar e levantar da cama (A), comer (B), pentear o cabelo (C), andar no plano (D), tomar banho (E), vestir-se $(F)$, ir ao banheiro em tempo sem que haja perda urinária ou fecal $(G)$, subir um lance de escadas $(H)$, medicar-se na hora (I), andar perto de casa $(\mathrm{J})$, fazer compras $(\mathrm{K})$, preparar refeições $(\mathrm{L})$, cortar as unhas dos pés $(M)$, sair da condução $(N)$, e fazer limpeza de casa $(0)$, respectivamente. Pode-se constatar que houve melhora das atividades deitar e levan-

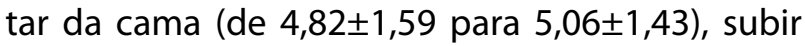

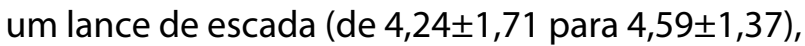
andar perto de casa (de 4,94 $\pm 1,25$ para $5,29 \pm 1,21$ ), fazer compras (de $4,24 \pm 1,86$ para $4,47 \pm 1,81$ ), sair da condução (de 4,35 $\pm 1,90$ para $4,82 \pm 1,88$ ) e fazer limpeza de casa (de 2,35 $\pm 2,26$ para 2,94 22,01 ), porém elas não apresentaram diferença estatisticamente significante. Da mesma forma, as atividades comer (de $6,00 \pm 0,00$ para $5,88 \pm 0,49$ ), pentear o cabelo (de 5,88 $\pm 0,49$ para $5,53 \pm 0,87$ ), vestir-se (de $5,18 \pm 1,42$ para $4,94 \pm 1,25$ ), preparar refeições (de $5,41 \pm 1,54$ para $5,06 \pm 2,01$ ) e cortar as unhas do pé (de 2,82 $\pm 2,24$ para $2,12 \pm 2,18$ ) apresentaram declínio pós-intervenção sem significância estatística. A única atividade com melhora estatisticamente significante após a intervenção foi a descrita como andar no plano (de 4,71 $\pm 1,21$ para 5,65 $\pm 0,79$ com $p=0,03$, respectivamente nas avaliações pré e pós intervenção), o que demonstra o benefício do programa aplicado.

Constatou-se que, de forma geral, houve manutenção de todas as atividades descritas ao se comparar os dados pré e pós-intervenção.

\section{DISCUSSÃO}

Observa-se a maior presença de mulheres no presente estudo que pode ser explicada pela maior preocupação que as idosas têm com relação a sua saúde ${ }^{22}$ e, conseqüentemente, à maior busca de atendimento médico ${ }^{23}$ e pelo fato de que a expectativa de vida das mulheres idosas é maior que 
dos homens, na qual para 100 mulheres idosas há menos que 81,6 homens idosos ${ }^{24}$.

Tabela 1. Comparação das médias de cada atividade pré e pósintervenção fisioterapêutica e a significância do estudo.

\begin{tabular}{|l|l|l|l|}
\hline Atividades & Pré & Pós & $p$ \\
\hline A- Deitar/Levantar da cama & $4,82 \pm 1,59$ & $5,06 \pm 1,43$ & 0,53 \\
\hline B - Comer & $6,00 \pm 0,00$ & $5,88 \pm 0,49$ & 0,33 \\
\hline C - Pentear o cabelo & $5,88 \pm 0,49$ & $5,53 \pm 0,87$ & 0,08 \\
\hline D - Andar no plano & $4,71 \pm 1,21$ & $5,65 \pm 0,79$ & 0,03 \\
\hline E - Tomar banho & $4,94 \pm 1,43$ & $4,94 \pm 1,43$ & 1,00 \\
\hline F - Vestir-se & $5,18 \pm 1,42$ & $4,94 \pm 1,25$ & 0,49 \\
\hline G - Ir ao banheiro em tempo & $5,41 \pm 0,94$ & $5,41 \pm 0,94$ & 1,00 \\
\hline H - Subir escada (1 lance) & $4,24 \pm 1,71$ & $4,59 \pm 1,37$ & 0,50 \\
\hline I - Medicar-se na hora & $5,53 \pm 1,12$ & $5,53 \pm 1,12$ & 0,38 \\
\hline J - Andar perto da casa & $4,94 \pm 1,25$ & $5,29 \pm 1,21$ & 0,33 \\
\hline K - Fazer compras & $4,24 \pm 1,86$ & $4,47 \pm 1,81$ & 0,60 \\
\hline L - Preparar refeições & $5,41 \pm 1,54$ & $5,06 \pm 2,01$ & 0,42 \\
\hline M - Cortar as unhas dos pés & $2,82 \pm 2,24$ & $2,12 \pm 2,18$ & 0,21 \\
\hline N - Sair de condução & $4,35 \pm 1,90$ & $4,82 \pm 1,88$ & 0,16 \\
\hline O - Fazer a limpeza de casa & $2,35 \pm 2,26$ & $2,94 \pm 2,01$ & 0,26 \\
\hline
\end{tabular}

Com relação à freqüência e à duração total do programa de tratamento, este estudo se apresenta coerente com os achados da literatura que fazem uso dessa mesma freqüência (duas vezes semanais) e referem melhora da variável dependente $^{9,10}$ e de duração mínima de dez semanas de duração deve ser utilizado para se obter eficácia na promoção de melhora das AVD's em uma intervenção25. Quanto ao tempo total da intervenção, o estudo presente necessitava de sessenta minutos para a realização de cada sessão ${ }^{26}$.

O protocolo proposto se assemelha com muitos descritos na literatura com relação ao uso de exercício de aquecimento ${ }^{27}$, de alongamento e resistência de grupos musculares antigravitacionais, pois estes estão intimamente relacionados com a execução das AVD's' ${ }^{26,27}$, de equilíbrio ${ }^{17}$, usando ponto de movimentação de quadril (já que toda reação de equilíbrio se inicia pela pelve), superfícies diferentes e caminhada já que intervenções que empreguem a caminhada e atividades de equilíbrio tentam harmonizar todo o sistema de controle do equilíbrio, além dos exercícios de relaxamento ${ }^{16,27}$. Mesmo assim a atividade andar no plano foi a única que apresentou melhora significante, provavelmente por causa do forte direcionamento dos exercícios do protocolo aos membros inferiores.
Os achados são diferentes dos relatados em outro estudo onde dez semanas de um programa de exercício fisioterapeutico composto por alongamento, fortalecimento muscular, atividade aeróbica e relaxamento resultou em melhora significativa no desempenho funcional com $p<0,01^{22}$. São diferentes também de outro estudo que apontou além de satisfação em realizar, com fisioterapeutas, os exercícios resistidos com foco em grupo musculares importantes no desempenho das AVD's, também melhora de força muscular e desempenho fucional pelo teste ADAP (Assessment of daily activity performance) com $p=0,001^{26}$.

Outro estudo com resultado divergente que refere melhora significativa da função mesmo após sete meses de intervenção nos idosos maiores que 75 anos de idade que não eram capazes de levantar-se da cadeira com os braços cruzados e que tivessem pontuação menor que 10 s no teste de caminhada rápida ${ }^{20}$. No entanto eles fizeram uso de exercícios aeróbicos, de orientações quanto à nutrição e de administração medicamentosa além dos de fortalecimento.

Uma hipótese para que não fosse evidenciada a melhora da atividade funcional com significância estatística em várias atividades é do baixo número de participantes que fizeram parte do presente estudo. $\mathrm{E}$ as atividades descritas como comer, pentear o cabelo, vestir-se, preparar refeições e cortar as unhas dos pés apenas apresentaram manutenção provavelmente porque não havia exercícios para membros superiores no conteúdo do programa e estas atividades avaliadas necessitam do uso deles.

Outra questão apontada é o fato de que, para avaliar as AVD's, só foram utilizados questionários de auto-relato e eles não expressam, com vigor, a realidade vivida pelos idosos. E um questionário de auto-relato combinado com a observação da atividade funcional poderia maximizar a descrição e avaliação do padrão da performance de uma atividade em questão, como já relatado na literatura ${ }^{28}$.

De qualquer forma, mesmo durante o período de tratamento, os participantes teriam declínio funcional relacionado aos fatores intrínsecos do envelhecimento e decorrentes da inativida$\mathrm{de}^{1,4,8,13,15,26}$. Já que isso é fato, a própria manutenção da performance já apresenta benefício para este grupo. 


\section{CONCLUSÃO}

Conclui-se que um programa de Fisioterapia baseado em exercícios de flexibilidade, de força e de equilíbrio resulta em manutenção de atividades funcionais da população idosa hígida. Novos trabalhos devem ser feitos associando os exercícios deste protocolo com o uso de exercícios para membros superiores.

\section{REFERÊNCIAS BIBLIOGRÁFICAS}

1.Savioli F, Ghorayeb N, Luiz CCC. Atleta idoso. In: Ghorayeb N. O exercício. Preparação fisiológica, avaliação médica, aspectos especiais e preventivos. São Paulo: Atheneu, 1999, 387-91.

2.Timo-laria C, Irigoyen C, Krieger EM. Fisiologia do envelhecimento. In: Petroianu A. Clínica e cirurgia geriátrica. Rio de Janeiro: Guanabara Koogan, 1999, 54-8.

3.Alves RV, Mota J, Costa MCC, Alves JGB. Physical fitness and elderly health effects of hydrogymnastics. Rev Bras Med Espor 2004;10:38-43. 4.Perrin P, Gauchard G, Perrot C, Jeandel C. Effects of physical and sporting activities on balance control in elderly people. $\mathrm{Br} F$ Sports Med 1999;33:121-6.

5.Nied R, Franklin B. Promotion and prescribing exercise for the elderly. Am Far Physician 2002;65:419-26.

6.Yuaso DR, Sguizzatto GT. Fisioterapia em pacientes idosos. In: Papaléo M. Gerontologia. A velhice e o envelhecimento em visão globalizada. São Paulo: Atheneu, 2002, 331-47.

7.Laughton C, Salvin M, Katdare K, Nolan L, Bean J, Kerrigan DC, et al. Aging muscle activity, and balance control: physiologic changes associated with balance impairment. Gait Post 2003;1-8.

8.Marks R, Allegrante J, MacKenzie CR, Lane J. Hip fracture among the elderly: cases, consequences and control. Aging 2003;2:57-93.

9.Seguin R, Nelson ME. Os benefícios do treinamento de força em adultos. Am J Preven Med 2003;25:141-9.

10.Skelton DA, Beyer N. Exercise and injury prevention in older people. Scand J Med Sci Sports 2003;13:77- 85.

11.Borst SE. Interventions for sarcopenia and muscle weakness in older people. Age aging 2004;33:548-55.

12.Deschenes MR. Effects of ageing on muscle fibre type and size. Sports Med 2004;33(12):809-24.

13.Boulgarides LK, McGinty S, Willett J, Barnes C. Use of clinical and impairment - based testes to predict falls by community - dwelling older adults. Phys Ther 2003;83(4):328-39.
14.Gardner MM, Robertson MC, Campbell AJ. Exercise in preventing falls related injuries in older people: a review of randomised controlled trials. Br F Sports Med 2000;34:7-16.

15.Campbell AJ. Preventing fractures by preventing falls in older women. CMAL 2002;167(9):1005-6.

16.Frank JS, Aftab E, Patla AE. Balance and mobility challenges in older adults. Implications for preserving community mobility. Am J Prevent Med 2003;25(3Sii):157-63.

17.Brawley LR, Rejeski J, King A. Promover atividade física para idosos. Os desafios para mudanças comportamentais. Am J Prevent Med 2003;25(3Sii):172-83.

18. Hatch J, Gill-Body KM, Portney LG. Os determinantes da confiança do equilíbrio nos moradores idosos de uma comunidade. Phys Ther 2003;83(12):1072-9.

19.Biderman A, Cwikel J, Fries AV, Galinsky D. Depression and falls among community dwelling elderly people: a search for common risk factors. J Epidemiol Comm Health 2002;56:631-6.

20.Gill Y, Baker D, Gottschlk M, Peduzzi P, Allore H, Ness P. A prehabilitation program for the prevention of functional decline: effect on highter-level physical function. Arch Phys Rehabil 2004;85:1043-9.

21.Blay LS, Ramos RL, Mari J. Validity of a Brazilian Version of the Older Americans Resources and Services (OARS) Mental Health Screening Questionnaire. JAGS 1988;36:687-92.

22.Salmela L, Santos L, Goulart F, Cassiano J, Hirochi T. Efeitos de atividades físicas e terapêuticas em adultos maduros e idosos. Fisioter Bra 2001;12(2):99-106.

23.Michel JP, Robine JM. A "new" general theory of population ageing. Gen Pap Risk Insur 2004;29:665-7.

24.Camargos MCS, Perpétuo IHO, Machado CJ. Expectativa de vida com incapacidade funcional em idosos em São Paulo. Rev Panam Salud Pública 2005;17:5-6.

25.Jensen J, Lundin-Oisson L, Nyberg L, Gustafson Y. Fall and injury prevention in older people living in residential care facilities. Aging Clin Exp Res 2004;16:283-92.

26.Vreede P, Samson M, Meeteren N, Bom JV, Duursma S, Verhaar H. Functional tasks exercise versus resistance exercise to improve daily function in older women: a feasibility study. Arch Phys Med Rehabil 2004;85:1952-61.

27.Santos LD, Same la LFT, Lelis FO, Lobo MB. Eficácia da atividade física na manutenção do desempenho funcional do idoso. Fisioter Bra 2001;2(3):169-77.

28.van Swearingen JM, Brach J. Making geriatric assessment works. Selecting useful measures. Phys Ther 2001;81:1233-52. 